\title{
The Extreme Ultraviolet Imager of Solar Orbiter: Optical Design and Alignment Scheme
}

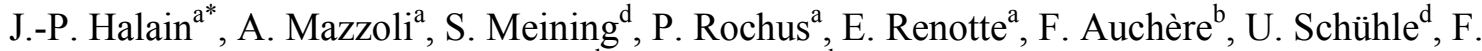

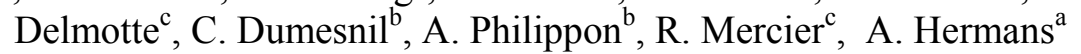 \\ ${ }^{a}$ Centre Spatial de Liège, Université de Liège, Liege Science Park, 4013 Angleur, Belgium \\ ${ }^{\mathrm{b}}$ Institut d'Astrophysique Spatiale, Orsay, France \\ 'Institut d'optique, Orsay, France \\ ${ }^{\mathrm{d}}$ Max-Planck-Institut für Sonnensystemforschung, Göttingen, Germany
}

\begin{abstract}
The Extreme Ultraviolet Imager (EUI) is one of the remote sensing instruments on-board the Solar Orbiter mission. It will provide dual-band full-Sun images of the solar corona in the extreme ultraviolet (17.1 nm and $30.4 \mathrm{~nm})$, and highresolution images of the solar disk in both extreme ultraviolet $(17.1 \mathrm{~nm})$ and vacuum ultraviolet (Lyman-alpha 121.6 $\mathrm{nm})$.

The EUI optical design takes heritage of previous similar instruments. The Full Sun Imager (FSI) channel is a singlemirror Herschel design telescope. The two High Resolution Imager (HRI) channels are based on a two-mirror optical refractive scheme, one Ritchey-Chretien and one Gregory optical design for the EUV and the Lyman-alpha channels, respectively.

The spectral performances of the EUI channels are obtained thanks to dedicated mirror multilayer coatings and specific band-pass filters. The FSI channel uses a dual-band mirror coating combined with aluminum and zirconium band-pass filters. The HRI channels use optimized band-pass selection mirror coatings combined with aluminum band-pass filters and narrow band interference filters for Lyman-alpha.

The optical performances result from accurate mirror manufacturing tolerances and from a two-step alignment procedure. The primary mirrors are first co-aligned. The HRI secondary mirrors and focal planes positions are then adjusted to have an optimum interferometric cavity in each of these two channels. For that purpose a dedicated alignment test setup has been prepared, composed of a dummy focal plane assembly representing the detector position.

Before the alignment on the flight optical bench, the overall alignment method has been validated on the Structural and Thermal Model, on a dummy bench using flight spare optics, then on the Qualification Model to be used for the system verification test and qualifications.
\end{abstract}

Keywords: Extreme Ultraviolet Imager, Solar Orbiter, Optical Design, Optical Alignment, Multilayer Coatings, Lymanalpha, Bandpass Filters

\section{INTRODUCTION}

The Extreme Ultraviolet Imager (EUI) instrument ${ }^{[6][10][13][14]}$ is one of the ten scientific instruments of the Solar Orbiter mission ${ }^{[1],[2],[3],[12]}$ dedicated to the observation of the Sun's atmosphere and heliosphere.

The EUI instrument is composed of two units, an Optical Bench System (OBS) and a Common Electrical Box (CEB) ${ }^{[6]}$. The OBS unit holds three telescope channels:

- $\quad$ A High Resolution Imager (100 km resolution) at the hydrogen Lyman- $\alpha$ line ${ }^{[9]}\left(\mathrm{HRI}_{\mathrm{Lya}}\right.$ channel),

- A High Resolution Imager (100 km resolution) at the extreme ultra-violet (EUV) $174 \AA$ line (HRI $\mathrm{I}_{\mathrm{EUV}}$ channel),

- A Full Sun Imager (900 km resolution) at alternatively the EUV $174 \AA$ and $304 \AA ̊$ lines ${ }^{[4],[5]}$ (FSI channel).

The performances of these telescopes result from accurate mirror manufacturing tolerances but also from a dedicated accurate alignment and co-alignment process. 


\section{OPTICAL DESIGN}

\subsection{FSI channel}

The FSI optical design is a one-mirror off-axis Herschelian telescope (Figure 2) with a $3.8 \mathrm{deg}$ x 3.8 deg FOV and an effective focal length of $462.03 \mathrm{~mm}$ (Figure 2) ${ }^{[14]}$.

A thin film aluminum filter is positioned $195 \mathrm{~mm}$ behind the entrance pupil. This filter rejects the visible light and the IR, letting only the EUV through.

The $174 \AA$ and $304 \AA$ FSI wavebands are selected using narrow bandpass filters and a dedicated multilayer coating of the mirror (Figure 1) optimized for high reflectance at both wavelengths ${ }^{[11]}$.

Two types of bandpass filters are mounted on a filter wheel, to alternatively select 174 or 304 A. They are multilayers of $\mathrm{Al} / \mathrm{Zr} / \mathrm{Al}$ (aluminum / zirconium / aluminum) and of $\mathrm{Al} / \mathrm{Mg} / \mathrm{Al}$ (aluminum / magnesium / aluminum).

The detector is a $3 \mathrm{k} \times 3 \mathrm{k}$ of $10 \mu \mathrm{m}$ pitch array, back-side passivated for EUV sensitivity.

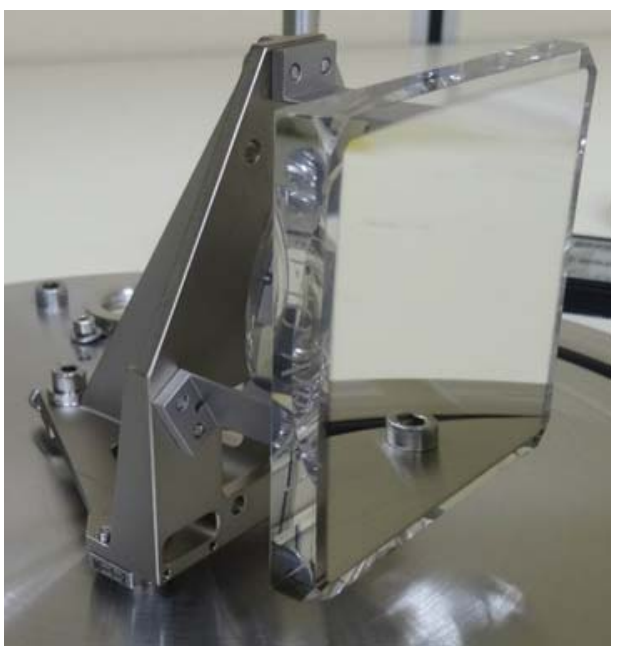

Figure 1 - FSI flight mirror with its multilayers coating

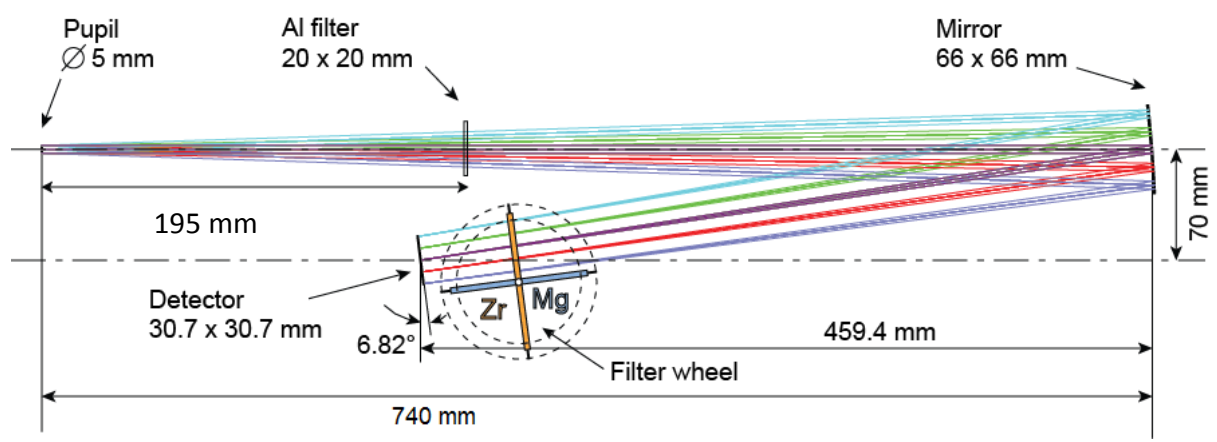

Figure 2 - The optical scheme of the FSI channel

Table 1 - Design parameters of the FSI channel

\subsection{HRI channels}

\begin{tabular}{|l|l|}
\hline Optical element & FSI \\
\hline Entrance pupil & Shape: hexagonal, 5 mm edge \\
\hline Primary mirror (M1) & $\begin{array}{l}\text { Figure: off-axis ellipsoid, conic }=-0.732 \\
\text { Shape: square } 66 \times 66 \mathrm{~mm} \\
\text { Off-axis: } 70 \mathrm{~mm} \text { - Focal length: } 462.5 \mathrm{~mm}\end{array}$ \\
\hline Distance Pupil - M1 & $740 \mathrm{~mm}$ \\
\hline Distance M1 - Focal plane & $462.03 \mathrm{~mm}$ (along optical axis) \\
\hline Detector & $\begin{array}{l}\text { Figure: flat square } 30.72 \times 30.72 \mathrm{~mm} \\
\text { Pixels: } 3072 \times 3072 \\
\text { Tilt to optical axis : } 6.82^{\circ}\end{array}$ \\
\hline
\end{tabular}

The $\mathrm{HRI}_{\text {Lya }}$ channel is an off-axis Gregory telescope, and the HRI $\mathrm{EUV}_{\mathrm{E}}$ channel is an off-axis Cassegrain telescope ${ }^{\text {[9][14] }}$. Both channels have been optimized in length and width, for the spacecraft volume and mass constraints, with a $30 \mathrm{~mm}$ and a $47.4 \mathrm{~mm}$ diameter entrance pupil, respectively, located at the front section of their entrance baffles (Figure 3 ). The optical design parameters are detailed in Table 2. 
Table 2 - Design parameters of the $\mathrm{HRI}_{\mathrm{EUV}}$ and $\mathrm{HRI}_{\mathrm{Ly}-\alpha}$ channels

\begin{tabular}{|c|c|c|}
\hline Optical element & HRI $_{\text {EUV }}$ & HRI $_{\text {Ly- } \alpha}$ \\
\hline Focal length & $4187 \mathrm{~mm}$ & $5804 \mathrm{~mm}$ \\
\hline Entrance pupil & $\phi 47.4 \mathrm{~mm}$ & $\phi 30 \mathrm{~mm}$ \\
\hline Field of view & 1000 arcsec square & 1000 arcsec square \\
\hline Plate scale & $50 \mathrm{arcsec} / \mathrm{mm}$ & $31.5 \operatorname{arcsec} / \mathrm{mm}$ (w/o intensifier taper) \\
\hline Primary mirror (M1) & $\begin{array}{c}66 \mathrm{~mm} \text { diameter (54 mm useful) } \\
80 \mathrm{~mm} \text { off-axis } \\
\mathrm{R}=1518.067 \mathrm{~mm} \mathrm{CC}, \mathrm{K}=-1\end{array}$ & $\begin{array}{c}42 \mathrm{~mm} \text { diameter (36 mm useful) } \\
80 \mathrm{~mm} \text { off-axis } \\
\mathrm{R}=1143 \mathrm{~mm} \mathrm{CC}, \mathrm{K}=-1\end{array}$ \\
\hline Secondary mirror (M2) & $\begin{array}{c}25 \mathrm{~mm} \text { diameter }(12 \mathrm{~mm} \text { useful }) \\
11.44 \mathrm{~mm} \text { off-axis } \\
\mathrm{R}=256.774 \mathrm{~mm} \mathrm{CX} \\
\mathrm{K}=-2.04\end{array}$ & $\begin{array}{c}20 \mathrm{~mm} \text { diameter (18 mm useful) } \\
7 \mathrm{~mm} \text { off-axis } \\
\mathrm{R}=91 \mathrm{~mm} \mathrm{CC} \\
\mathrm{K}=-0.65\end{array}$ \\
\hline
\end{tabular}

For the HRI $\mathrm{EUV}_{\mathrm{V}}$ channel, one aluminum foil filter is inserted between the entrance aperture (entrance pupil) and the primary mirror to provide protection against excessive heat input on the mirror and efficient rejection of the visible light. A filter wheel, comprising two redundant EUV filters, one open and one occulting position, is located at the output pupil. The front and rear EUV filters are of primary importance for the instrument to suppress the visible light that can be $10^{8}$ times more intense than the EUV flux. The EUV reflective coatings of the mirrors are specific multilayers optimized to provide the desired scientific spectral passband. Their design takes into account the angle of incidence on the mirrors, which variations are small enough so that no compensation is needed.

The detector of the $\mathrm{HRI}_{\mathrm{EUV}}$ channel is also $3 \mathrm{k}$ x $3 \mathrm{k}$ of $10 \mu \mathrm{m}$ pitch array, back-side passivated for EUV sensitivity. Only the central $2 \mathrm{k}$ x $2 \mathrm{k}$ window is however used.

The Lyman- $\alpha$ channel mirrors use $\mathrm{MgF}_{2} / \mathrm{Al}$ (magnesium fluoride/ aluminum) coating providing a reflectivity at $121.6 \mathrm{~nm}$ over $75 \%$. A broad-band $\mathrm{MgF}_{2}$ interference filter is used at the entrance of the telescope to isolate the spectral line at $121.6 \mathrm{~nm}$ and reject visible and infrared light as well as EUV and X-rays, protecting the mirror coatings. A narrow-band filter is placed in front of the detector to further isolate the Lyman- $\alpha$ line and achieve the spectral purity. The combination with a solar-blind detector yields a spectral purity larger than $90 \%$ for Lyman- $\alpha$ in the quiet Sun and higher purity in the active regions, and is tolerant to potential spectral shifts due to thermal effects. The magnesium fluoride substrate material of the filters is sufficiently radiation hard so as to provide the thermal heat load protection with minimal degradation during the mission.

The detector of the Lyman- $\alpha$ channel will be a solar-blind, intensified $2 \mathrm{k}$ x $2 \mathrm{k}$ CMOS active pixel sensor (I-APS) with a sensitive aperture of $32 \times 32 \mathrm{~mm}$. The image of the intensifier is transferred by a fibre optic taper to the actual size of the CMOS/APS sensor providing an image scale of 1 arcsec on two pixels.

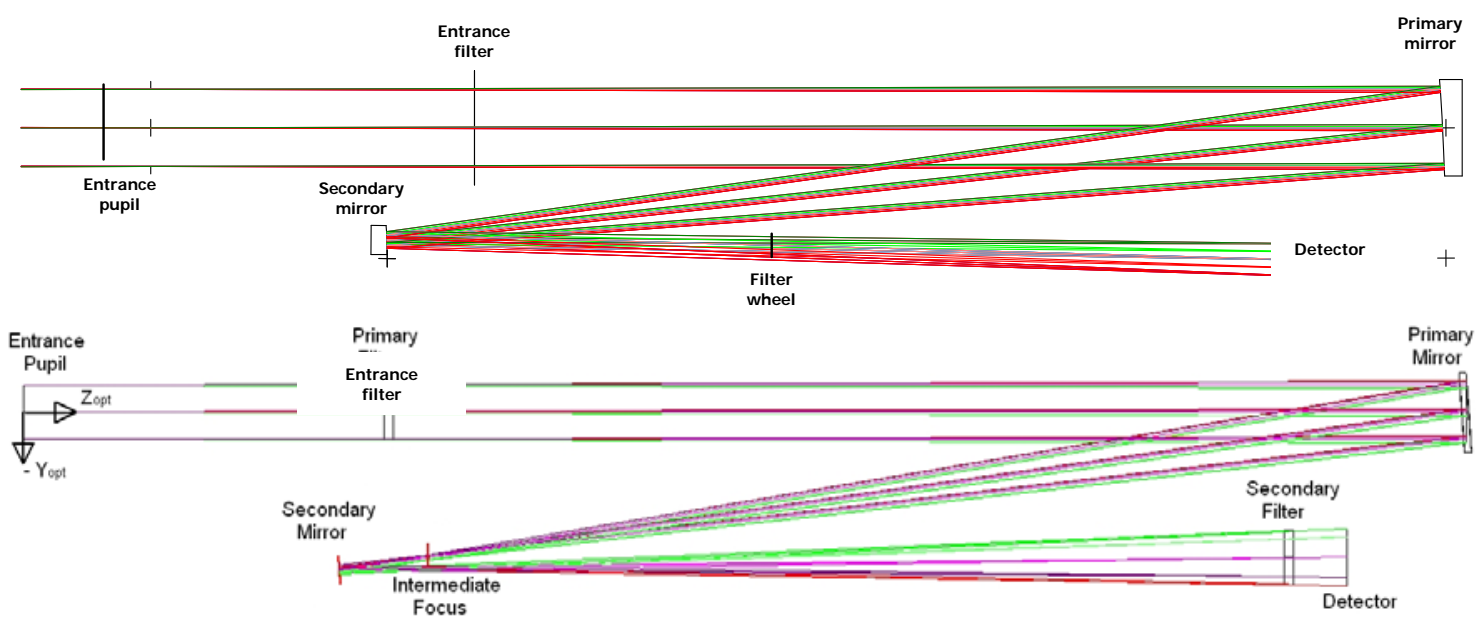

Figure 3 - EUI HRI optical layouts (top: $\mathrm{HRI}_{\mathrm{EUV}}$ channel, bottom: $\mathrm{HRI}_{\mathrm{Lya}}$ channel) 


\section{OPTICAL ALIGNMENT}

The optical alignment of the EUI instrument is performed in two major steps:

- A co-alignment of the channels line of sights $(\mathrm{LoS})$ with the OBS main reference cube (which is itself co-aligned with the $\mathrm{S} / \mathrm{C}$ master reference cube) to ensure the three telescopes have the same pointing on the Sun. The coalignment between channels and main reference cube is obtained by adjusting the primary mirrors tilts.

- An interferometric alignment of the HRI channels. By use of the secondary mirrors and detector position adjustment capabilities, an optimum interferometric cavity is obtained in each of these two HRI channels. A dummy camera is used to measure the detector optimum position, which is then reported within the flight cameras.

\subsection{Unit reference cubes}

The OBS unit is equipped with two reference cubes, one located on the $X$ (front) side and one on the $+X$ (back, non-flight) side of the unit.

The co-alignment of these two cubes is obtained by mechanical tolerances of their support fixation on the bench, resulting however in a residual small but constant offset.

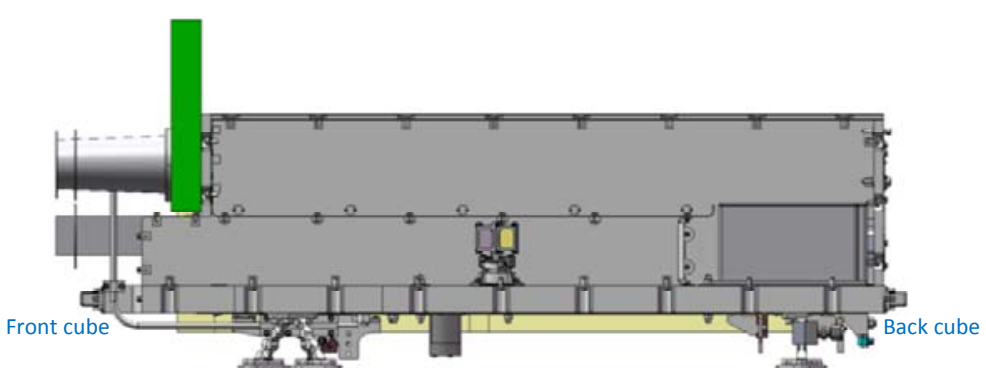

Figure 4 - EUI OBS front $(-\mathrm{X})$ and back $(+\mathrm{X})$ reference cubes

\subsection{Alignment template}

A template supports the instrument during the alignment. It mechanically and optically materialise the optical bench optical axis and its reference frame, and has the same mounting interface with the instrument than the spacecraft (three interface pads for the instrument feet and a dowel pin at the URF hole). It comprises a master reference cube to set the instrument optical axis w.r.t. the template, and 3 reference balls to set the instrument mechanical axis w.r.t. the template.
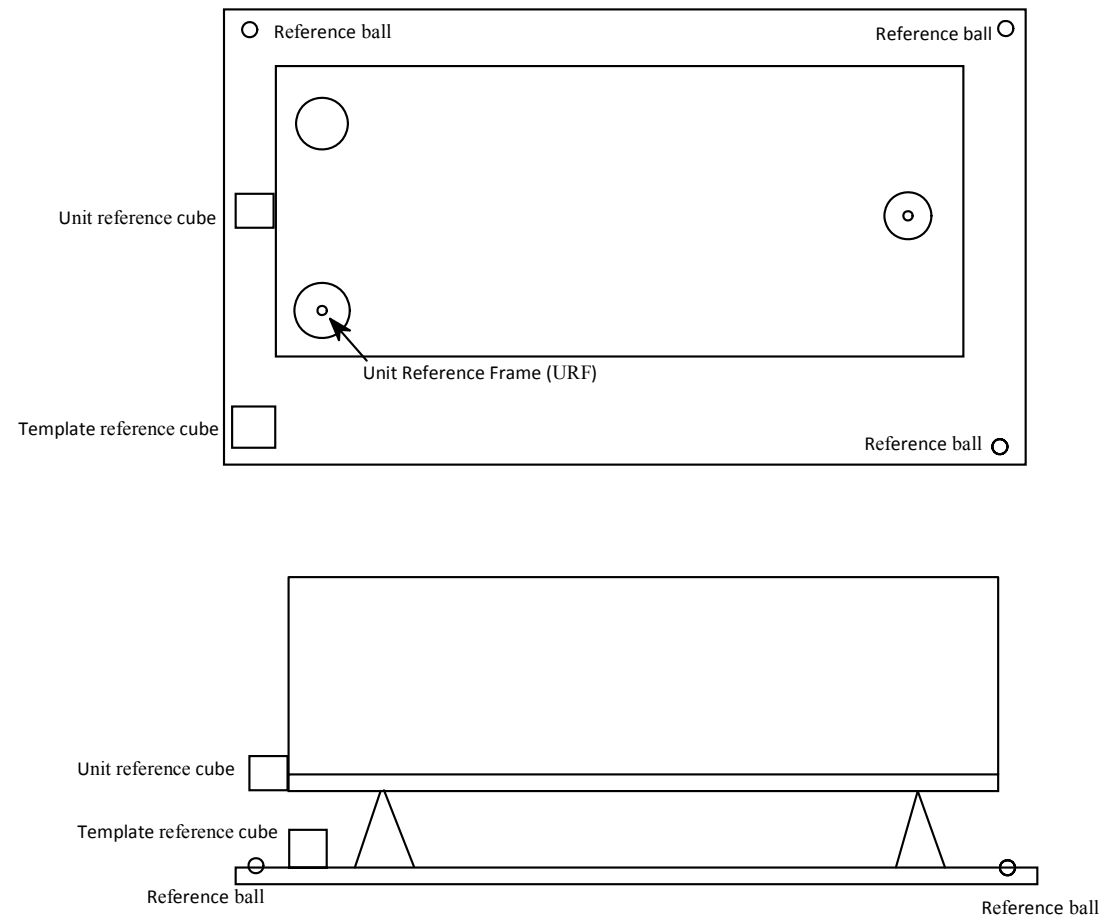

Figure 5 - EUI OBS front $(-\mathrm{X})$ and back $(+\mathrm{X})$ reference cubes 


\subsection{Dummy bench}

In order to validate the co-alignment and interferometric alignment, a dummy bench with the same mechanical interfaces for mirrors and cameras is used. This bench allows practicing and optimising the method in parallel with the other OBS unit activities (mechanism assembly, bake-out...) and limits the risks associated with flight hardware manipulation.
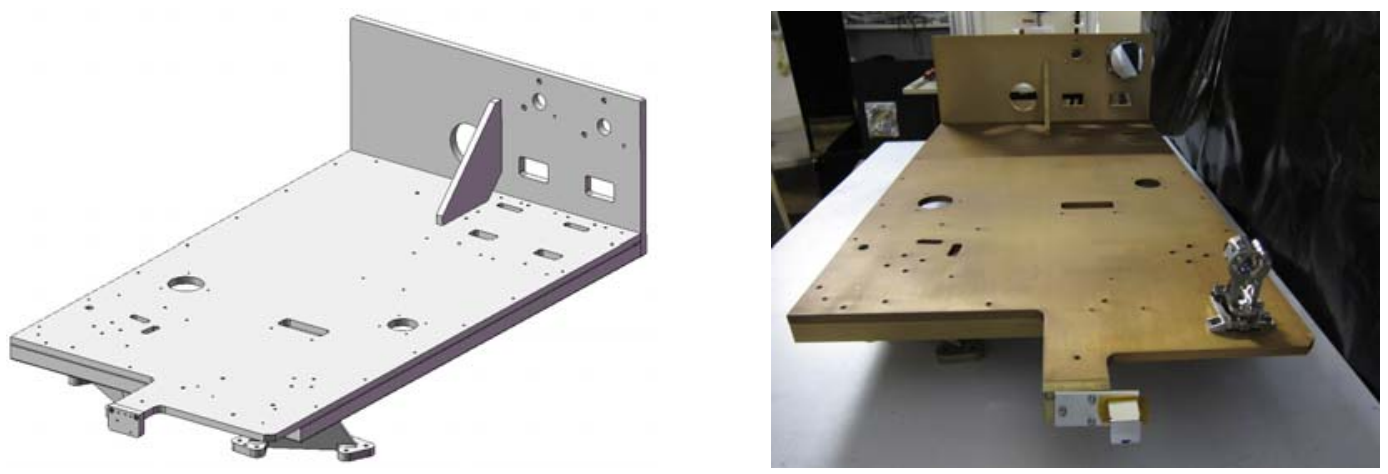

Figure 6 - EUI OBS dummy bench used for alignment practice and optimisation

\subsection{Channels co-alignment}

The co-alignment of the channels line of sights $(\mathrm{LoS})$ is mandatory to ensure that they point to the same region of the Solar disk.

The co-alignment is performed by adjusting the primary mirror of each channel to be co-aligned with the OBS reference cube. The expected orientation errors of the primary mirrors reference surface w.r.t. the mirrors optical axis is $<10$ arcsec. The co-alignment error between the channels is then expected to be $<30$ arcsec, and the expected orientation error of the OBS reference cube w.r.t. the OBS mechanical axis is $<30 \mathrm{arcsec}$.

It is planned to check the co-alignment of the three channels by using a common collimated light source illuminating the three channels and by identifying the illuminated pixel on each channel detector.

\subsection{STM activities}

During the instrument Structural and Thermal Model (STM) activities ${ }^{[14]}$, a repetition of the channel co-alignment was performed. The objectives were also o verify the mirror mount stability during environmental tests (before/after vibrations, and during thermal balance test) and assess the bench thermo-elastic stability (during thermal balance test) with the thermo-elastic structural analysis.

Flat mirrors (on flight representative mounts) were however used instead of flight-shape representative mirrors. The flat mirror angles indeed allowed auto-collimation with theodolites, as shown in Figure 7. For the FSI channel, which has one mirror, a flat reflective surface was located as dummy detector. The co-alignment was performed using adjustment of HRI secondary mirrors / FSI dummy detector orientation.

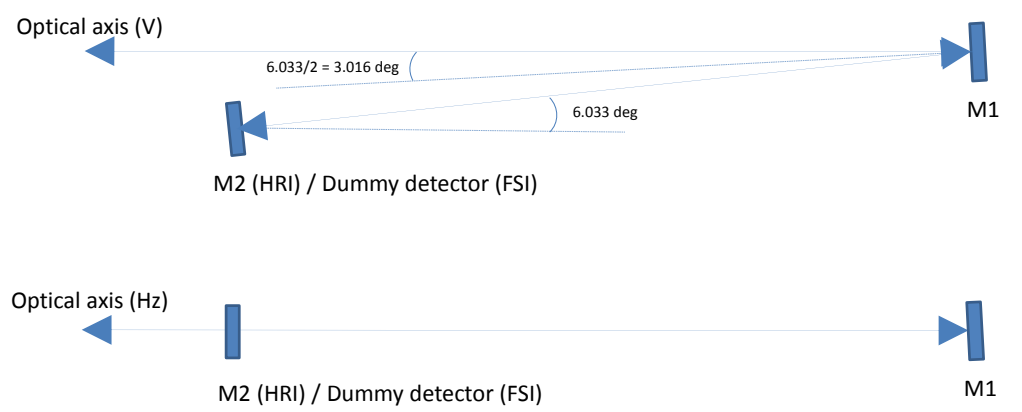

Figure 7 - STM optical scheme, with flat mirror used for co-alignment check (vertical - V, and Horizontal - Hz) 
The STM co-alignment was performed according to the following steps.

- The reference cubes (V and Hz) angular offset was measured.

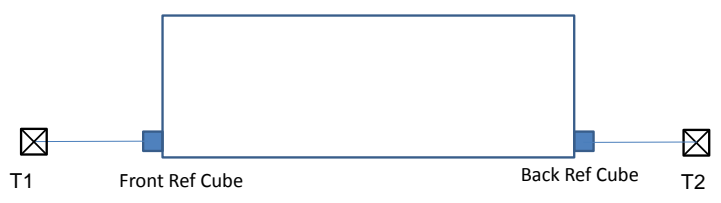

- The HRI channel primary mirrors (M1) offset is measured vs. the back reference cube, using M1 back surface (which is parallel to front surface).

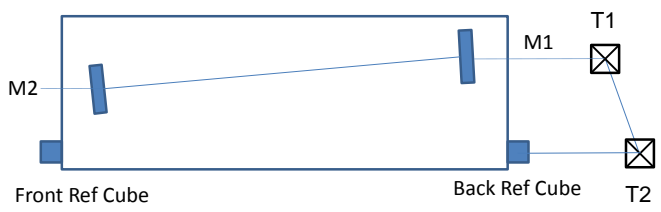

- The HRI channel secondary mirrors (M2) offset are measured vs. the front reference cube, and M2 are adjusted to adjust the optical axis (OA) parallel with the front reference cube (by use of autocollimation through M1 and M2).

- The FSI channel optical axis vs. the reference cube is measured (by use of auto-collimation through M1 and a reflective dummy detector).
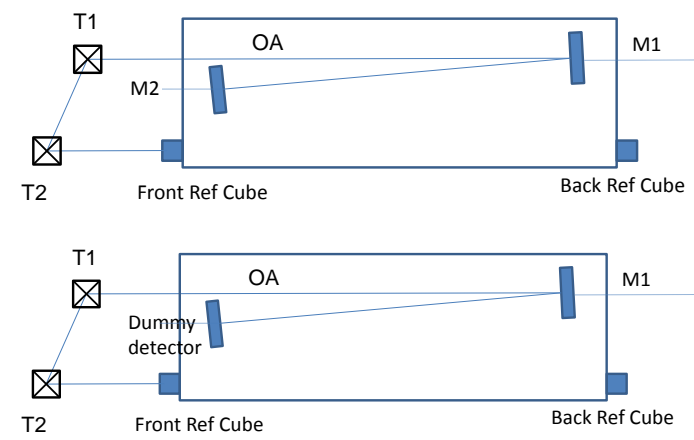

Once the channels were co-aligned, the STM has passed mechanical and thermal test and co-alignment check was performed at the end.

From thermal tests, the main outcome is given on Figure 8 (left). It shows a $40-50$ arcsec evolution of the HRI $\mathrm{EUV}_{\mathrm{E}}$ primary mirror tilt, per $20^{\circ} \mathrm{C}$ overall temperature variation, while it shall be smaller than 30 arcsec over the operational temperature range of -20 to $+50^{\circ} \mathrm{C}$ (i.e. $70^{\circ} \mathrm{C}$ excursion). This measurement was confirmed using both front and back sides of the HRI primary mirror.
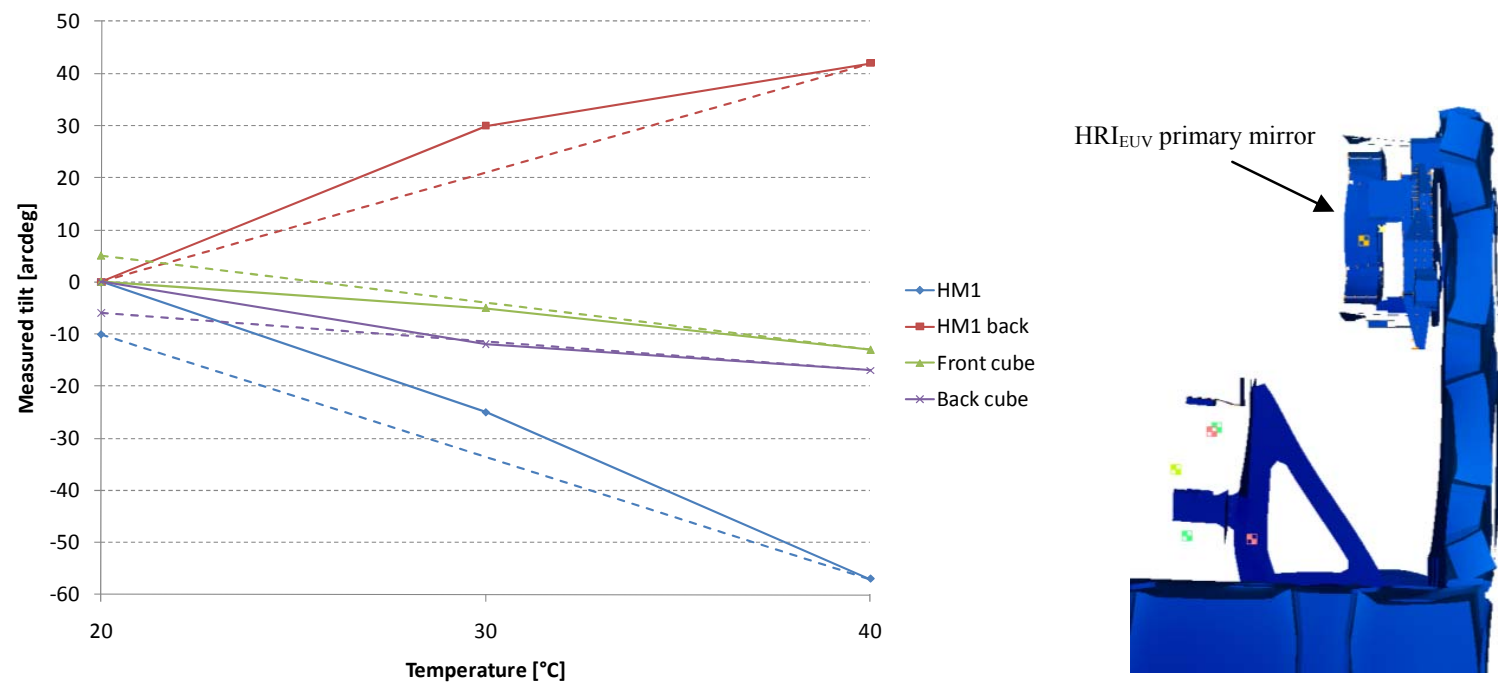

Figure 8 - Left: EUI HRI $\mathrm{EUv}_{\text {p }}$ primary mirror (HM1) tilt evolution over $20^{\circ} \mathrm{C}$ temperature range. Right: Thermo-elastic Model of the HRI $\mathrm{EUV}_{\mathrm{E}}$ mirror on the OBS structure back panel. 
A detailed thermo-elastic model of the back panel (Figure 8 - right) confirmed that the bench thermoelastic stability was not sufficient to guarantee the primary mirror stability over the thermal range.

Reinforcement of the OBS structure, and in particular of its back panel (on which these mirrors are mounted) was necessary and implemented for the flight model to reduce the mirror tilts with temperature.

From mechanical tests, the main outcome is the confirmation of the optical axis stability. The offset between the front and back reference cubes was measured after each random test. Figure 9 shows a variation of the vertical (V) and horizontal $(\mathrm{Hz})$ offsets of respectively 30 and 5 arcsec (lower than the specified 1 arcmin).

\subsection{HRI interferometric alignment}

Once on the flight optical bench, after the channel co-alignment with the optical bench reference cube, the interferometric alignment of each HRI channel is based on the following steps:

1. The mirror characteristics are measured after manufacturing (WFE, vertex position, curvature...), as shown for example for the $\mathrm{HRI}_{\mathrm{EUV}}$ primary mirror on Figure 10, and used to update the optical model and derive optimum position/orientation of the secondary mirror.
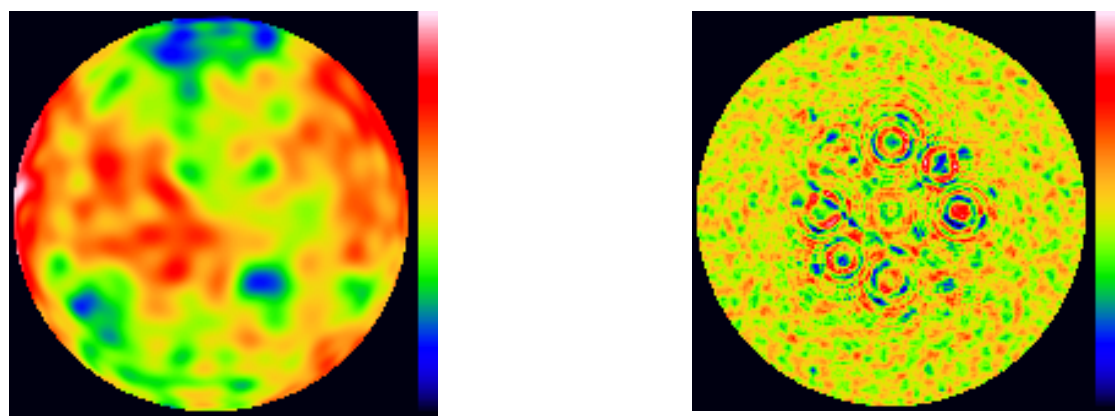

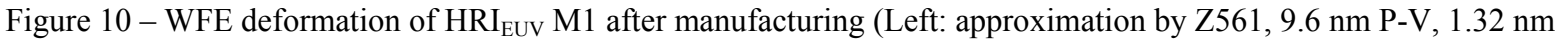
RMS. Right: residual defects, $4.6 \mathrm{~nm}$ P-V, $40 \mathrm{~nm}$ over $99.9 \%$ pixels, $0.45 \mathrm{~nm}$ RMS)

2. A dummy Focal Plane Assembly (FPA) is set on the optical bench, instead of the real camera, allowing to adjust the position of the focal plane (i.e. position of detector within the real camera), as shown on Figure 11.

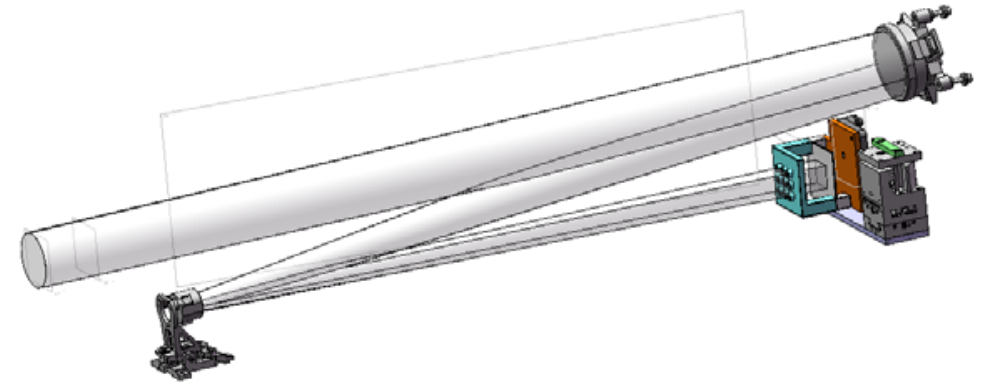

Figure 11 - HRI channel configuration with primary and secondary mirrors, and dummy FPA 
3. The secondary mirror is set on the optical bench according to the optimised position and orientation, and the interferometric cavity is optimised (i.e. spot size is reduced) by adjusting the secondary mirror and the focal plane position/orientation as shown on Figure 12. Table 3 list the parameters that can be used for this interferometric alignment (and for the channel co-alignment).

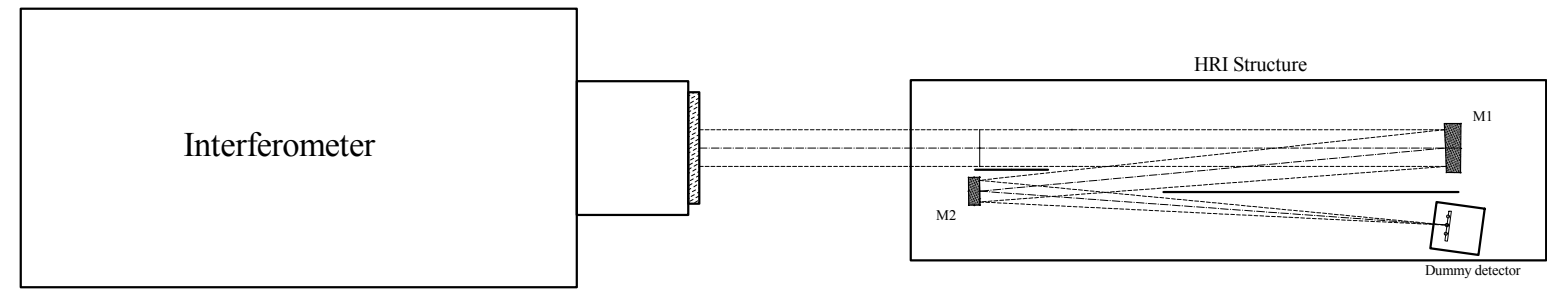

Figure 12 - Configuration for interferometric measurement

Considering the mirrors manufacturing errors, alignment errors and setting errors, the RMS WFE (in one pass) for zero FOV is about $\lambda / 40$ at $\lambda=633 \mathrm{~nm}$ for a PV WFE around $\lambda / 10$. As a consequence the interferometer test plate surface quality of $\lambda / 20$ should be good enough to perform interferometric measurements. In addition, as the HRI entrance pupil diameter (47.4 $\mathrm{mm}$ for $\mathrm{HRI}_{\mathrm{EUV}}$ and $30 \mathrm{~mm}$ for $\mathrm{HRI}_{\mathrm{Lya}}$ ) is smaller than the test plate diameter (100 $\mathrm{mm})$, this test plate surface quality on that smaller diameter must be better than $\lambda / 20$. Test plates with a $\lambda / 40$ surface quality are available and will improve the measurements accuracy. Figure 13 shows this WFE in one pass at $\lambda=633 \mathrm{~nm}$.

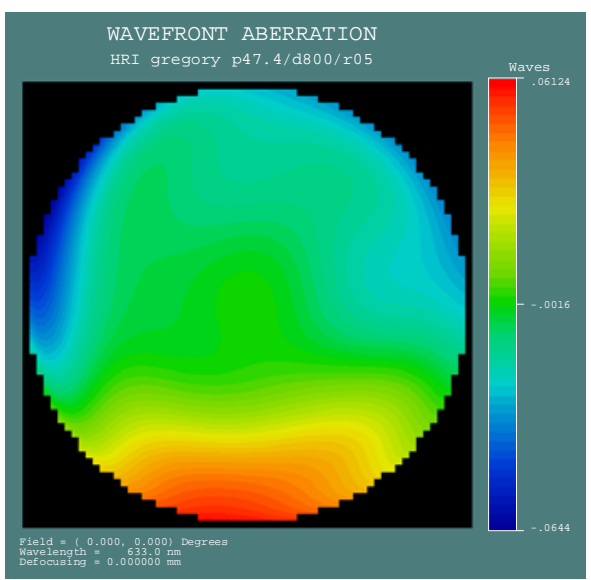

Figure 13: WFE in one pass, with mirrors manufacturing, alignment and setting errors at $633 \mathrm{~nm}$

4. When the alignment is optimized, the detector is set within the real camera at the same position than the dummy focal plane.

5. The camera with real detector is set on the optical bench and is used to cross-check the alignment using a collimated light beam or a theodolite.

Table 3 - Adjustment parameters for the HRI channel interferometric alignment and co-alignment

\begin{tabular}{|l|c|c|c|}
\hline Item & Tip/tilt & Focus & Translation \\
\hline Primary mirror (M1) & $\mathrm{X}$ & $\mathrm{X}$ & \\
\hline Secondary mirror (M2) & $\mathrm{X}$ & $\mathrm{X}$ & $\mathrm{X}$ \\
\hline Dummy FPA & & $\mathrm{X}$ & \\
\hline
\end{tabular}

\subsection{Dummy FPA}

The dummy FPA is composed of two parts with different purposes:

- The first part is a set of lenses used for the interferometric alignment of the mirrors.

- $\quad$ The second part is a set of pinholes used to check the optical axis position and the distortion. 
For the interferometric alignment only the central lens is used, the other lenses are used after the alignment to check the WFE in the FOV. The dummy FPA with the lenses can be easily removed and replaced during the alignment so that the WFE measurement and optical axis check can be alternated.

The pinhole plate is composed of 17 pinholes corresponding to the central FOV, intermediate FOV and maximum FOV. The pinholes have a diameter of 10 $\mu \mathrm{m}$ (with $2 \mu \mathrm{m}$ accuracy in diameter). The accuracy on the pinholes position is $3 \mu \mathrm{m}$ w.r.t. the central pinhole.

This plate is backlight illuminated and used to be pointed through the channel by a theodolite (in direct view mode, not in auto-collimation).

The dummy FPA has the same interface than the real FPA so that the real FPA can be positioned at the exact same place as the dummy FPA after the alignment.

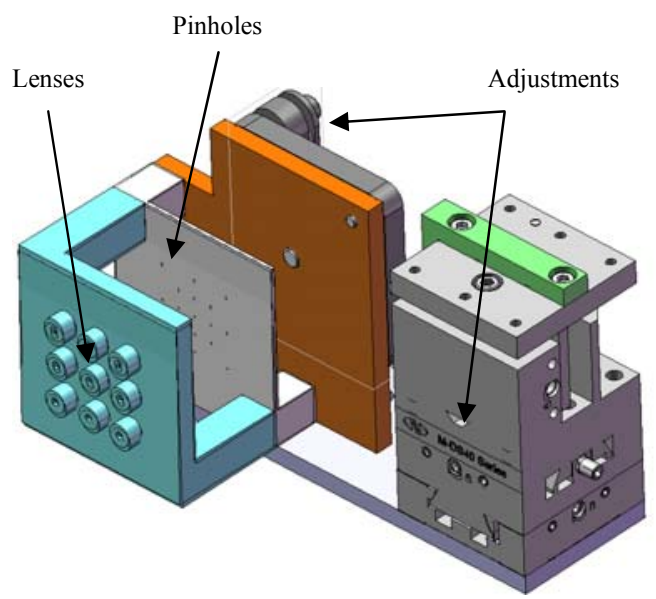

Figure 14 - Dummy FPA used for EUI channel alignment

\subsection{FSI channel adjustment}

The FSI channel should be within tolerances without interferometric alignment and its alignment is much less critical than the two HRI channels. The mirror being fixed once co-aligned with the unit reference cube (by use of shims under the mirror, $100 \mu \mathrm{m}$ shim being equivalent to 10 pixel shift on detector), the only remaining parameter is the focus allowing to fine tune the spot size. The HRI dummy FPA is thus used to verify the alignment and optimise the detector (and mirror) focus. For the mirror focus, a fine tuning is possible with custom screw threads. Table 4 lists the FSI mirror adjustment parameters as per Figure 15.

Table 4 - Adjustment parameters of the FSI mirror

\begin{tabular}{|l|c|c|c|c|}
\hline & Fabrication & Adjustment & Range & Increment \\
\hline $\boldsymbol{\Delta X}$ & $\pm 0.1 \mathrm{~mm}$ & No & - & - \\
\hline $\boldsymbol{\Delta Y}$ & $\pm 0.1 \mathrm{~mm}$ & Yes & $\pm 0.1 \mathrm{~mm}$ & $<0.01 \mathrm{~mm}$ \\
\hline $\boldsymbol{\Delta Z}$ (focus) & $\pm 0.1 \mathrm{~mm}$ & Yes & $\pm 0.5 \mathrm{~mm}$ & $<0.06 \mathrm{~mm}$ \\
\hline $\boldsymbol{\theta X}$ & $\pm 0.8^{\prime}$ & Yes & $\pm 3.4^{\prime}$ & $<40^{\prime}$, \\
\hline $\boldsymbol{\theta Y}$ & $\pm 1.35^{\prime}$ & No & - & - \\
\hline $\boldsymbol{\theta Z}$ & $\pm 1.0^{\prime}$ & Yes & $\pm 3.4^{\prime}$ & $<40^{\prime}$, \\
\hline
\end{tabular}

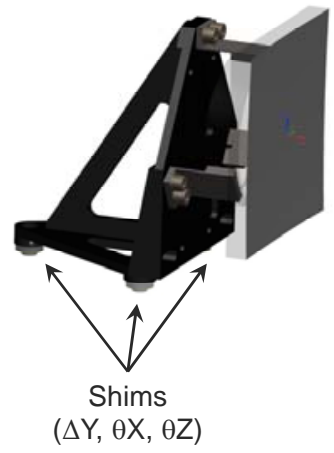

Figure 15 - FSI mirror adjustments

\section{CONCLUSIONS}

The EUI instrument is a three channel telescope that requires both co-alignment of its channels and interferometric alignment of its two high-resolution channels.

The co-alignment has been practiced and successfully achieved on the STM unit. It also served to assess the thermoelastic stability of the optical bench, leading to improvement of this structure to ensure the required stability over the operational temperature range.

The interferometric alignment will now be first repeated on a dummy bench in advance of the qualification and then the flight structure. For that purpose a dummy focal plane, used to determine the detector best position and orientation, has been manufactured and will be used sequentially for each channel. 


\section{ACKNOWLEDGEMENTS}

The EUI instrument is developed in a collaboration which includes the Centre Spatial de Liège (Belgium), the Institut d'Astrophysique Spatiale and the Institut d'Optique (France), the UCL Mullard Space Science Laboratory (UK), the Max Planck Institute for Solar System Research (Germany), the Physikalisch-Meteorologisches Observatorium Davos (Switzerland), and the Royal Observatory of Belgium (Belgium).

The Belgian institutions are funded by Belgian Federal Science Policy Office (BELPSO); the French institutions by Centre National d'Etudes Spatiales (CNES); the UK institution by the UK Space Agency (UKSA); the German institution by Deutsche Zentrum für Luft- und Raumfahrt e.V. (DLR), and the Swiss institution by the Swiss Space Office (SSO).

\section{REFERENCES}

[1] Fleck B., Harrison R. A., Marsden R. G., Wimmer-Schweingruber R., "Summary of the Solar Orbiter payload working group activities, Telescopes and Instrumentation for Solar Astrophysics" Proc. SPIE 5171, 123-130, (2004).

[2] Marsden R.G., Marsch E. and the Solar Orbiter Science Definition Team, "Solar Orbiter Science Requirements Document" SCI-SH/2005/100/RGM, Issue 1 Revision 2 (2005).

[3] Mc Coy D., and the Solar Orbiter assessment team, "Solar Orbiter Payload Definition Document" SCI$\mathrm{A} / 2004 / 175 / \mathrm{AO}$, Issue 5 Revision 0 (2006).

[4] Rochus P., Halain J.P., Renotte E., Berghmans D., Zhukov A., Hochedez J.F., Appourchaux T., Auchère F., Harra L.K, Schühle U., Mercier R.., "The Extreme Ultraviolet Imager (EUI) on-board the Solar orbiter Mission" 60th International Astronautical Congress, (2009).

[5] Hochedez J.-F., Appourchaux T., Defise J.-M., Harra L. K., Schuehle U., Auchère F., Curdt W., Hancock B., Kretzschmar M., Lawrence G., Marsch E., Parenti S., Podladchikova E., Rochus P., Rodriguez L., Rouesnel F., Solanki S., Teriaca L., Van Driel L., Vial J.-C., Winter B., Zhukov A., "EUI, The Ultraviolet Imaging Telescopes of Solar Orbiter" The Second Solar Orbiter Workshop, (2006).

[6] Halain J.-P., Rochus P., Appourchaux T., Berghmans D., Harra L., Schühle U., Auchère F., Zhukov A., Renotte E., Defise J.-M., Rossi L., Fleury-Frenette K., Jacques L., Hochedez J.-F., Ben Moussa A., "The technical challenges of the Solar-Orbiter EUI instrument" Proc. SPIE 7732, 26 (2010)

[7] Halain J.-P. , Berghmans D., Defise J.-M., Renotte E., Thibert T., Mazy E., Rochus P., Nicula B., De Groof A., Seaton D., Schühle U., "The First light of SWAP on-board PROBA2" Proc. SPIE 7732, 24 (2010)

[8] Auchère F., et al., "HECOR, a HElium CORonagraph aboard the Herschel sounding rocket" Proc. SPIE 6689, (2007)

[9] Schühle U., Halain J., Meining S., Teriaca L., "The Lyman-alpha telescope of the extreme ultraviolet imager on Solar Orbiter" Proc. SPIE Solar Physics and Space Weather Instrumentation IV, 8148, (2011)

[10] Halain J.-P., Rochus P., Renotte E., Appourchaux T., Berghmans D., Harra L., Schühle U., Schmutz W., Auchère, F., Zhukov A., Dumesnil C., Kennedy, T., Mercier R., Pfiffner D., Rossi L., Tandy J., Smith P., "The EUI instrument on board the Solar Orbiter mission: from breadboard and prototypes to instrument model validation" Proc. SPIE 8443, (2012)

[11] Delmotte F., et al., "Development of multilayer coatings for Solar Orbiter EUV imaging telescopes" Proc. SPIE 8877, (2013)

[12] Fahmy S., Bagnasco G., Pacros A., Wirth K., "Solar Orbiter payload suite: a hotbed of innovation" 64th International Astronautical Congress, IAC-13-A3.5.2, (2013)

[13] Halain JP, Debaize A., Gillis JM., Jacques L., De Ridder T., Hermans L., Koch M., Meynant G., Schippers G., "The dual-gain $10 \mu \mathrm{m}$ back-thinned 3k x 3k CMOS-APS detector of the Solar Orbiter Extreme UV Imager” Proc. SPIE, 9144, (2014)

[14] Halain JP, Rochus P, Renotte E, Auchère F, Berghmans D, Harra L, Schühle U, Schmutz W, Zhukov A, Aznar Cuadrado R, Delmotte F, Dumesnil C, Gyo M, Kennedy T, Mercier R, Verbeeck C, Thome M, Heerlein K, Hermans A, Jacques L, Mazzoli A, Meining S, Rossi L, Tandy J, Smith P, Winter B, "The Extreme UV Imager of Solar Orbiter - From detailed design to Flight Model" Proc SPIE, 9144-7, (2014) 